\title{
Transatlantica
}

Revue d'études américaines. American Studies Journal

\section{Anti-conservateur, anti-radical, pro-militant : Richard Rorty, ou la séparation de la philosophie et de l'Etat}

David Barral

\section{(2) OpenEdition}

Journals

Édition électronique

URL : http://journals.openedition.org/transatlantica/3053

DOI : 10.4000/transatlantica.3053

ISSN : $1765-2766$

Éditeur

AFEA

Référence électronique

David Barral, « Anti-conservateur, anti-radical, pro-militant : Richard Rorty, ou la séparation de la philosophie et de l'Etat », Transatlantica [En ligne], 1 | 2008, mis en ligne le 14 mai 2008, consulté le 29 avril 2021. URL : http://journals.openedition.org/transatlantica/3053 ; DOI : https://doi.org/10.4000/ transatlantica.3053

Ce document a été généré automatiquement le 29 avril 2021.

\section{(c) (i) (9)}

Transatlantica - Revue d'études américaines est mis à disposition selon les termes de la licence Creative Commons Attribution - Pas d'Utilisation Commerciale - Pas de Modification 4.0 International. 


\title{
Anti-conservateur, anti-radical, pro- militant : Richard Rorty, ou la séparation de la philosophie et de l'Etat
}

\author{
David Barral
}

Liste des abréviations pour les ouvrages de Richard Rorty :

PSH : Philosophy and Social Hope (1999)

AOC : Achieving Our Country (1999)

ORT : Objectivity, Relativism, and Truth (1991)

1 Parmi les accueils les moins favorables réservés en France au pragmatisme du philosophe américain Richard Rorty, il en est un qui souligne parfaitement le problème qui va nous occuper ici, celui de la relation (ou de la non-relation) entre philosophie et politique. Dans Recommencer la Philosophie : La philosophie américaine aujourd'hui, Sandra Laugier parle des «limites du relativisme culturel de Rorty [qui] semble mener tout droit à un hyperconformisme, irréfutable de surcroît » (Laugier, 54). Un peu plus loin, on peut lire la phrase suivante: «il n'y a rien dans l'œuvre de Rorty [...] qui soit réellement en connexion avec la culture et l'histoire de l'Amérique dans ce qu'elles ont d'original » (Laugier, 55). Un jugement si lapidaire appelle à l'évidence la question suivante : si la philosophie de Richard Rorty aboutit à une défense politique du statu quo, et si la tradition de pensée dont il se réclame n'a rien de "véritablement américain ", ce philosophe n'est-il pas doublement exclu d'une réflexion collective sur le thème de «L'Amérique militante »?

2 Nous pensons que non, et allons donc tenter de montrer ce que la pensée de Rorty peut apporter quand on s'interroge sur le militantisme aux Etats-Unis. Pour être plus précis, nous allons considérer la situation de Rorty au sein de la polémique qui a occupé, et occupe encore, une grande partie du débat public contemporain outre-Atlantique, c'est-à-dire la controverse qui oppose la gauche universitaire et la droite réactionnaire, 
les intellectuels radicaux et les intellectuels conservateurs, sur les questions philosophiques et culturelles de la vérité objective et des valeurs. Mais avant tout, nous voudrions définir, même brièvement et de façon très incomplète, les positions philosophique et politique de Rorty.

Quant à la philosophie, Rorty est un pragmatiste, qui se réclame de la lignée de William James $^{1}$ et de John Dewey ${ }^{2}$ (PSH, 23-39). Politiquement, c'est un partisan du libéralisme, qui se réclame toujours de l'héritage de John Dewey (AOC, 3-38).

4 L'adjectif «pragmatiste" recouvre un certains nombre de positions d'ordre philosophique, et plus précisément épistémologique (c'est-à-dire qui relèvent de la théorie de la connaissance et, par extension, de la théorie de la vérité). Nous pouvons résumer ces positions ainsi : il n'existe pas de connaissance ou de vérité indépendantes du langage, de la culture, de la conceptualisation et de l'interprétation humaines, donc pas de connaissance ou de vérité qui soit au-delà de l'histoire ou de la culture. Autrement dit, il n'y a ni connaissance ni vérité objective, ou absolue (PSH, «Introduction: Relativism: Finding and Making », xvii-xviii). Voilà pour la position philosophique de Rorty, dont nous pouvons dire que les principes sont globalement partagés par les universitaires d'extrême gauche, ces théoriciens de la postmodernité dont nous allons bientôt parler.

5 Pour ce qui est de l'étiquette politique « libéral », la meilleure façon de la comprendre est d'utiliser la problématique de John Rawls, un des plus grands théoriciens américains de la justice : «comment faire pour que subsiste, dans le temps, une société stable et juste de citoyens libres, égaux, et profondément divisés par des doctrines religieuses, philosophiques et morales différentes? " (Rawls, «Introduction », xxvii) Le libéralisme répond à cette question en proposant qu'aucun recours ne soit fait à des conceptions métaphysiques ou épistémologiques particulières dans le règlement des affaires publiques : cette position est donc non seulement laïque, partisane d'une séparation entre l'Eglise et l'Etat à la Jefferson, mais aussi "antifondationnaliste ", c'est-à-dire partisane d'une séparation entre les absolus de la philosophie et le domaine de la politique. La "Vérité Objective», pas plus que la religion ou toute autre doctrine morale ou philosophique, ne peut prétendre, dans le contexte d'une démocratie pluraliste, à régler la vie de tous les citoyens.

6 Pour résumer : le philosophe Rorty pense qu'il n'y a pas de vérité hors contexte ; mais le citoyen, ou le penseur politique en lui pense que sa position philosophique ne peut pas plus être le fondement de nos pratiques politiques qu'une position philosophique adverse, ou que la théologie.

7 Aujourd'hui, de nombreux historiens des idées politiques (Christopher Lasch, James Kloppenberg et Benjamin Barber, pour ne mentionner qu'eux) s'accordent sur le diagnostic suivant: dans le cadre des "guerres culturelles» (culture wars) auxquelles se livrent conservateurs et radicaux, le pragmatisme de Rorty est très souvent confondu avec son libéralisme, et le philosophe constitue la cible privilégiée d'attaques provenant des deux côtés de l'échiquier politique. Le témoignage autobiographique du critique Stanley Fish (qui n'accepterait cependant sans doute pas l'épithète « libéral ») nous renseigne sur la teneur de ces attaques : «à droite, la critique consiste à dire qu'en défendant [l'idée que] l'interprétation est inévitable, je défais la trame de la civilisation et j'ouvre la voie à l'anarchie nihiliste. L'objection venant de la gauche est que je ne le fais pas assez $»^{4}$ (Fish, 307). Rorty est l'objet d'accusations identiques, comme il l'explique au début d'un essai autobiographique (" Trotsky and the Wild Orchids », PSH, 
3-4). Pour les néo-conservateurs, sa philosophie équivaut à nier la morale et à saper la culture américaine ; pour leurs adversaires, il ne tire pas les conséquences politiques de sa position philosophique, attaché qu'il reste à la démocratie américaine (une certaine démocratie américaine, célébrée par Whitman) et à ses idéaux (AOC; $\mathrm{PSH}, 4)$. La schizophrénie n'est pas loin.

8 Afin de répondre à ces critiques, nous nous proposons, dans un premier temps, d'élucider simultanément deux questions : 1) pourquoi, aux Etats-Unis, le débat public contemporain est-il centré sur les notions de valeur et de vérité objective, plutôt que sur des questions économiques et sociales, comme c'était le cas avant les années soixante, ou en tout cas, avant la Seconde Guerre mondiale ? 2) Pourquoi le débat public est-il clivé de façon si catégorique, avec d'un côté les théoriciens radicaux de la postmodernité et de l'autre, les conservateurs traditionalistes? Répondre à ces questions nous permettra d'expliquer le phénomène que Rorty appelle «l'éclipse de la gauche réformiste par la gauche culturelle » (AOC, 41-107).

9 Nous montrerons ensuite que conservateurs anti-universitaires et radicaux universitaires, malgré le fossé politique qui les séparent, partagent l'idée suivante : les théories de la vérité ont des implications politiques majeures. Selon les conservateurs, seule l'existence de vérités absolues rend légitimes nos systèmes politiques; selon les radicaux, l'absence de vérités absolues rend tout système illégitime. En réfutant les deux faces de cette même idée, c'est-à-dire en séparant la philosophie et l'Etat, le libéralisme de Rorty répond simultanément au conservatisme et au radicalisme universitaire.

10 Enfin, nous tâcherons d'expliquer en quoi le caractère anti-théorique de la position politique de Rorty peut-être qualifié de " pro-militant ».

De la gauche politique à la gauche culturelle : vers un débat politique bipolaire

11 Les "guerres culturelles» opposent, à droite, une sensibilité conservatrice et, à l'extrême gauche, une sensibilité radicale. La sensibilité libérale de Rorty ne constitue pas une troisième voix politique véritablement reconnue : pour toute reconnaissance, le libéralisme est soit taxé d'hégémonisme, soit d'anarchisme. Le débat est donc essentiellement bipolaire. Comment rendre compte de ce clivage extrêmement figé ? Une chose est sûre: quand la forme du débat public est moulée sur une alternative aussi étanche, tout ce qui ne rentre pas dans une des deux catégories passe au second plan, ou, pire, chez l'adversaire. Dans le cas qui nous occupe, la victime de la bipolarisation est une certaine gauche qui a traditionnellement voulu éviter la révolution comme la réaction: il s'agit de la gauche américaine socio-libérale, cette gauche réformiste associée à Dewey et au New Deal dont Rorty brosse le portrait dans Achieving Our Country. Dans l'analyse de Rorty (mais Kloppenberg et Lasch ne disent pas autre chose), le clivage " conservateurs-radicaux ", et la disparition de ce qu'on appelle " the Old Left " $(\mathrm{AOC}, 42)$, correspondent aux années soixante, et particulièrement à l'événement déterminant de la guerre du Vietnam. Le clivage du débat public contemporain trouve en effet son origine dans la déchirure tragique de l'opinion américaine sur la question de la guerre. Cette déchirure est d'autant plus douloureuse et persistante que la division politique se double d'une division générationnelle.

12 Afin d'illustrer ce point, nous allons utiliser un exemple tiré de la littérature contemporaine: le roman de Philip Roth, American Pastoral. Roth y expose les tourments d'un père de famille libéral dont la fille, une jeune radicale, pose, en guise de protestation à la guerre, une bombe qui fera un mort. La violence de l'histoire 
fictionnelle permet à Roth de dépeindre le phénomène suivant: la vieille école américaine du libéralisme est, à l'image du personnage du père (incarnation d'une gauche libérale, sociale et patriote à la Dewey), littéralement balayée par la haine qu'éprouve la nouvelle génération pour une nation qui peut s'engager militairement au Vietnam. Tout dialogue est rompu entre la génération des parents, qui croient aux idéaux fondateurs des Etats-Unis, et une jeunesse qui foule au pied ces idéaux. D'un côté, le père continue de croire à ses valeurs politiques, et à l'efficacité du recours institutionnel, et de l'autre, sa fille ne parle que de subvertir ces valeurs, et se lance dans la revendication radicale. S'opposent ainsi, d'un côté, l'approche réformiste du père, membre d'un comité d'hommes d'affaires du New Jersey contre la guerre, qui va jusqu'à Washington pour être reçu par un sénateur; et de l'autre, la violence meurtrière de sa fille. Il existe sur ce point une correspondance intéressante entre cette fiction et l'histoire des idées politiques, selon laquelle le mouvement américain contre la guerre du Vietnam a été un relatif échec, non pas à cause d'un consensus en faveur de la guerre, mais bien à cause des méthodes de contestation employées par ses membres les plus radicaux. La cause entière aurait donc été discréditée par le caractère extrêmement violent ainsi que l'anti-américanisme patent d'une certaine opposition à la guerre (PSH, 260).

13 Cependant, comme le montre bien le roman de Roth, ce n'est pas simplement une cause ponctuelle en matière de politique étrangère, mais toute une façon de penser qui a été balayée par les "sixties» et le radicalisme estudiantin. Balayée parce que, bien évidemment, la violence symbolique de la notion de subversion totale des valeurs est fascinante. Et par la fascination qu'elle exerce, l'idée de subversion totale éclipse tout naturellement les discours plus modérés. Il est donc assez facile, dans ce cadre, de comprendre l'éclipse de la gauche réformiste, ainsi que la résurgence du conservatisme. James Kloppenberg en donne l'analyse suivante: "[l]es penseurs conservateurs [...] ont cherché à exploiter les angoisses suscitées par le mouvement des droits civiques, la New Left et le féminisme. [...] Quand les radicaux ont commencé à défier toute autorité politique et culturelle, à mépriser le civisme et à chanter les louanges du plaisir, quand ils se sont proclamés adeptes de la drogue, du sexe, et de la musique subversive, même des gens qui se considéraient comme des libéraux ont commencé à prendre peur ${ }^{5} »$ (Kloppenberg, 150-151).

14 Au-delà de l'opposition à la guerre du Vietnam, c'est donc bien cette volonté explicite de subvertir les valeurs traditionnelles d'un pays considéré comme totalement corrompu qui sert de tremplin politique au conservatisme. A la fin des années 70 , le débat public est devenu le lieu d'opposition entre la subversion postmoderne et la réaction traditionaliste. Le libéral, dans ce contexte, est alternativement stigmatisé comme le héraut de l'impérialisme ou le chantre de l'anarchie. Dans ce qui devient une guerre des valeurs culturelles, la question de la justice économique et sociale est reléguée au second plan. Rorty, dans Achieving Our Country, n'en rend pas moins hommage à la colère qui a secoué le pays de 1964 à 1972, sans laquelle, selon lui, les Etats-Unis auraient pu bien plus mal tourner. Il reconnait le fait que, si les appels à la révolution des années soixante ont, heureusement, été un échec, ils ont ranimé l'activisme de gauche (AOC, 68-69).

15 Mais la situation change dans les années soixante-dix et quatre-vingt, quand les étudiants des «Sixties » se retrouvent professeurs : cette génération d'universitaires a un goût prononcé pour ce que Rorty appelle la « philosophie apocalyptique française et 
allemande [de Foucault et Heidegger] » (AOC, 77). Avant les années soxiante, c'était le département des sciences sociales qui était le plus actif à l'Université (AOC, 77). Or, dans la nouvelle façon de pratiquer la critique sociale qui voit le jour dans les "Seventies», Marx est en partie remplacé par Freud, l'économie politique par la philosophie, et la question de l'argent par celle de l'«humiliation» (AOC, 76-81). Cela signifie que la revendication principale ne porte plus sur un égal accès aux moyens d'une vie réussie, considération presque exclusivement matérielle, mais concerne la reconnaissance et le respect, ce qui relève plus de la psychologie. Selon Rorty, ce changement dans l'ordre des priorités a eu le mérite suivant : ce que la droite a baptisé le "politiquement correct» a considérablement changé la façon dont la société américaine envisage les minorités ethniques et culturelles, et promu l'intégration (PSH, 260). Mais cela n'empêche pas Rorty de constater que le prix à payer pour cette réduction de l'humiliation est très élevé : «[1]es universitaires de gauche ont permis à la politique culturelle de supplanter la politique réelle, et ils ont collaboré avec la Droite afin de centrer le débat public sur des questions culturelles $»^{6}(\mathrm{AOC}, 14)$. La collaboration, à l'évidence, est inconsciente : il s'agit pour la droite et la gauche de tomber d'accord sur ce qui leur permet de s'opposer. Ce qui est remarquable, et qui doit faire l'objet d'une attention immédiate, c'est que la gauche universitaire et la droite conservatrice partagent la même conception des relations entre philosophie et politique - une conception que Rorty remet en cause.

Radicalisme universitaire et conservatisme anti-universitaire : deux camps politiques, une seule prémisse philosophique

Après l'activisme politique des années soixante, une partie de la gauche, dont les membres sont en général professeurs de littérature, devient donc « culturelle » (AOC, 75-107). Différents commentateurs, de sensibilités politiques opposées, lui donnent de nombreux autres noms : «Ecole du Ressentiment $~^{7}$ (Harold Bloom, cité in ORT, 179), « relativisme radical» (Cheney, 17), «gauche universitaire» (Lasch, 177), «pseudoradicalisme universitaire » (Lasch, 176), « scepticisme nihiliste » (Kimball, cité in Lasch, 187), « radicalisme culturel» (Kloppenberg, 10). Rorty, quand il souhaite en souligner les excès, l'appelle, à la suite d'Allan Bloom, « gauche nietzschéenne » (Nietzscheanized Left, cité in PSH, 129), et "gauche spectatrice» (spectatorial left, AOC, 38) quand il souhaite en souligner la froideur analytique. Selon lui, cette gauche est une anomalie aux Etats-Unis dans la mesure où elle ne croit pas à la capacité de rédemption ou d'amélioration que les institutions démocratiques devraient conférer à la société américaine $(\mathrm{AOC}, 38)$ : alors que la gauche américaine traditionnelle insistait sur une certaine loyauté envers les idéaux fondateurs de la nation, la "gauche nietzschéenne " explique à la nation qu'elle est "pourrie en son cœur - que c'est une société raciste, sexiste, impérialiste, [...] une société dont chaque discours doit être déconstruit sans ménagement $»^{8}(\mathrm{PSH}, 129)$.

Le fait que Rorty reprenne à son compte le qualificatif «nietzschéenne » n'est pas innocent, puisque cette gauche est "philosophique» ou, selon la formule de Rorty: "incroyablement obsédée par elle-même [...] et victime d'une passion pour la philosophie qui confine à l'absurde $»^{9}(\mathrm{PSH}, 129)$. La conception que le critique Paul de Man, chef de file de la déconstruction à l'américaine, se fait de la politique, est un bon exemple de cette passion: selon lui, celle-ci ne peut s'envisager que sous l'angle d'une déconstruction littéraire. Plus généralement, les radicaux universitaires partagent l'idée que l'action politique est essentiellement affaire de "problématisation de concepts familiers » $(\mathrm{AOC}, 93)$ et de « subversion » de ceux-ci. Il y a donc deux éléments 
qui rendent la gauche culturelle politiquement inopérante en dehors du cadre strictement universitaire : 1) le caractère excessivement théorique de ses écrits, qui implique une certaine forme d'élitisme intellectuel; et 2), son mépris pour la société américaine contemporaine. Sa seule véritable action, louable mais limitée au cadre universitaire, consiste à promouvoir la reconnaissance de cultures dites " alternatives " (afro-américaine, gay et lesbienne, par exemple) - ce qui se fait en intégrant de nouveaux auteurs au programme; et qui provoque, bien évidemment, l'indignation de la droite traditionaliste, Lynne Cheney à sa tête. Dans les années quatre-vingt et quatrevingt-dix le débat public le plus médiatique, entre droite et extrême gauche, a donc pour enjeu la transformation ou la conservation du canon des œuvres littéraires.

Cependant, malgré tout ce qui les oppose, droite conservatrice et gauche universitaire partagent l'idée suivante : s'il n'y a pas de fondations absolues à la vie politique d'une nation, alors c'est l'anarchie. Pour les deux camps, la théorie selon laquelle il n'y a pas de vérité hors-contexte a une conséquence majeure : rien de moins que la fin de la politique, et de la civilisation occidentale, telle que nous les connaissons. Bien sûr, les émotions que suscite cette conclusion ne sont pas les mêmes: inquiétude chez les conservateurs, contre jubilation chez les radicaux. D'où deux modes de réponses différents: d'un côté, les radicaux culturels se limitent à l'écriture d'ouvrages universitaires accomplissant la déconstruction conceptuelle de l'hégémonie W.A.S.P.; ils invoquent l'argument que toute connaissance est une construction idéologique, et donc que toutes les valeurs se valent. De l'autre, les conservateurs exhument et brandissent la notion d'objectivité ainsi que les valeurs transcendantes qui auraient un jour constitué les fondations de la civilisation américaine et occidentale. Autrement dit, «nihilisme » et "Vérité » deviennent leurs slogans respectifs. Les conservateurs, à l'instar de Lynne Cheney, William Bennett et Allan Bloom, célèbrent la "Vérité " comme fondation absolue, indépendante de l'esprit humain, qui seule peut garantir les pratiques politiques et protéger la nation contre le relativisme qui la ronge. Quant aux radicaux universitaires, conformément à leur interprétation de théoriciens comme Michel Foucault, ils glorifient le «nihilisme », et concluent au relativisme culturel, au multiculturalisme, et à l'anarchie : tout système politique est nécessairement illégitime. Car en l'absence de vérités absolues, tout n'est qu'idéologie; et si aucun critère objectif ne nous permet de choisir entre plusieurs systèmes idéologiques, comment et surtout pourquoi défendre la très arbitraire démocratie libérale à l'américaine?

Rorty ne pense pas qu'il faille un absolu pour légitimer les institutions politiques, ni que l'absence d'un tel absolu les rende illégitimes (ORT, 175-196). La notion que partagent conservateurs et radicaux, selon laquelle la théorie de la connaissance a des conséquences politiques, est aussi suspecte que le fait de fonder une politique étrangère ou intérieure sur la volonté de Dieu. Selon Rorty, la politique est à la fois beaucoup plus simple que ce que voudraient nous faire croire les abstractions théoriques des radicaux universitaires, et beaucoup plus compliquée que le recours conservateur à des vérités indépendantes de l'esprit humain. La politique n'est ni disqualifiée par la subjectivité, ni garantie par l'objectivité: dans nos sociétés démocratiques, c'est le lieu d'un dialogue entre personnes, donc le lieu de l'intersubjectivité. Radicaux et conservateurs prennent tout simplement beaucoup trop au sérieux les développements récents en philosophie, c'est-à-dire l'idée que la vérité est un produit humain et pas une correspondance à une réalité extra-humaine. Or pour Rorty, la philosophie n'est qu'une discipline universitaire qui n'est pas plus habilitée à fournir les règles du débat public que la théologie ou quelque autre secteur de la 
culture. Considérons la question suivante, une problématique très rawlsienne : entre toutes les doctrines philosophiques, morales et religieuses, laquelle doit-on choisir pour conduire le débat public? (Rawls, xx-lxii; 3-46). Les sceptiques radicaux répondent selon l'argument communautarien classique: "aucune, car toutes se valent »-fin de la discussion. Les conservateurs répondent : « les nôtres » - fin de la discussion également. Mais pour Rorty, et ceux qu'il appelle les libéraux, la politique n'est pas censée répondre à cette question. Ce que la politique doit garantir, c'est que la réponse à la question du choix de valeurs soit laissée à la discrétion de chaque citoyen, que cette réponse soit une affaire privée. Quant au débat public caractéristique des démocraties occidentales, il doit non pas arrêter mais perpétuer la discussion en vue d'un consensus sur les buts communs à poursuivre et les réformes à promouvoir - des buts et des réformes qu'il faut toujours réviser; le débat public ne doit pas être une lutte autour des valeurs que tous devraient adopter ou abandonner une fois pour toutes; il ne doit pas être moral et perfectionniste (dans l'acception philosophique du mot: «la recherche de la bonne vie »), mais doit garantir la poursuite individuelle de perfections morales différentes. Par où la position politique anti-théorique de Rorty peut être qualifiée de pro-militante.

Rorty, philosophe pro-militant : le retour à la gauche réformiste

20 Le cheval de bataille politique de Rorty est celui de la gauche libérale traditionnelle, c'est-à-dire la justice économique et sociale par le biais de réformes successives. Ce que reproche Rorty à la gauche culturelle, c'est d'avoir permis que le débat public soit pris en otage, depuis la fin des années 70, par des questions culturelles et théoriques plutôt que sociales et économiques. Cette configuration du débat arrange bien sûr les conservateurs. Selon l'histoire des idées politiques récente, la gauche radicale et théorique a été le "meilleur ennemi » de la droite conservatrice, en contribuant à reléguer la question des réformes économiques au second plan (AOC, 41-107; Kloppenberg, 3-37, 124-154). De plus, après l'activisme estudiantin des années soixante et du début des années soixante-dix, on ne peut plus parler de véritable militantisme politique au sein de la gauche culturelle: en se retranchant derrière les murs de l'Université et les publications universitaires, celle-ci est devenue polémique plutôt que militante. Délaissant l'action politique, elle se livre à une guérilla théorique. Comme le dit Todd Gitlin ${ }^{10}$, que Rorty paraphrase avec approbation : «nous autres universitaires avons envahi le département d'Anglais, pendant que les Républicains prenaient d'assaut la Maison Blanche ${ }^{11}$ (PSH, 260). La conséquence positive de cette invasion est, rappelons-le, l'instauration du " politiquement correct », dans sa version modérée, et la reconnaissance des cultures dites minoritaires ou alternatives. Mais la contrepartie en est l'abandon de ce que Rorty appelle "la politique de classe» (class politics, par opposition à academic politics) (PSH, 255-261) ou encore "politique réelle » (real politics) (AOC, 14), celle que veut pratiquer la gauche libérale. (Il convient sans doute de rappeler que la gauche et le radicalisme qui nous occupent ici sont ceux qui existent au sein de l'université : il ne s'agit donc pas, à l'évidence, ni pour Rorty ni pour nous qui le commentons, de nier l'existence et la permanence dans le temps de revendications provenant d'autre lieux que l'université, et du militantisme, de l'activisme qui les accompagnent; il s'agit en revanche de souligner la prédominance médiatique de la gauche culturelle, et de constater à quel point celle-ci peut nuire à la publicité d'un radicalisme plus proprement politique, et à un militantisme digne de ce nom). distinction entre «spectateur » et «acteur » $(\mathrm{AOC}, 105)$ : l'universitaire radical est le 
paradigme du spectateur de la société, qui, abstrait de celle-ci, dénonce ses travers; le réformiste libéral est le paradigme de l'acteur, engagé dans la transformation et l'annulation de ces travers. Notons qu'il est nécessaire, pour être spectateur, de se situer en dehors du spectacle que l'on observe: cette métaphore spatiale est parfaitement rendue dans la façon dont la gauche universitaire envisage sa situation: ses membres ne se considèrent absolument pas comme partie intégrante de la culture socio-politique démocratique américaine. Ne pouvant s'identifier à celle-ci, ils prétendent juger la société depuis leurs bastions universitaires ; cette attitude traduit, en l'occurrence, un désespoir total en les Etats-Unis, qui désamorce conséquemment toute volonté ou tentative de réforme. Pour le libéral, en revanche, l'espoir en l'amélioration de ces travers sert de motivation à la réforme. Selon l'analyse de Rorty, les théoriciens postmodernes qui ont lu Foucault et Heidegger considèrent que la citoyenneté américaine est quelque chose dont on ne peut pas être fier, et que toute participation à la politique électorale est vaine (AOC, 32-35). La gauche radicale a la conviction que les Etats-Unis ont perdu leur innocence à jamais ; qu'ils sont, dans leurs valeurs, leur culture et leurs institutions, corrompus au-delà de toute réforme. S'il est admis qu'en politique une dimension critique ou autocritique est bien loin d'être négligeable, il faut reconnaître que ce n'est pas tout. Or depuis les années soixante, aux Etats-Unis, le vocabulaire de la subversion a occulté celui de la réforme, la question de la culture a occulté celle de la justice économique et sociale, les abstractions de la théorie ont occulté les propositions concrètes de réforme, la polémique épistémologique a occulté le militantisme véritablement politique, et le désespoir en la nation a occulté le patriotisme nécessaire à toute gauche désirant rivaliser avec la droite sur le terrain de la politique électorale (AOC, 7).

C'est là que la position anti-théorique, anti-philosophique de Rorty se révèle promilitante: lorsqu'il suggère que le désespoir et l'excès théorique n'incitent pas au militantisme de gauche, c'est-à-dire à la promotion active de réformes ayant pour but l'égalité sociale et économique. Selon Rorty, il est urgent de faire une différence entre la polémique philosophique à laquelle s'est livrée la gauche culturelle avec la droite conservatrice, et le militantisme, la véritable action politique qu'une gauche patriote et réformiste avec un programme concret aurait au moins le mérite de rendre possible. La suggestion de Rorty, selon laquelle les théories postmodernes de la connaissance n'ont rien à voir avec la manière de pratiquer la politique, se veut donc une issue à ce que Christopher Lasch appelle "l'impasse universitaire»(Lasch, 14); une impasse philosophique dans laquelle s'est malheureusement fourvoyé le débat public contemporain, au détriment de toute discussion véritablement politique au premier plan. Notons qu'il relèverait du contresens de désigner Rorty comme un philosophe militant, puisque il n'appuie pas ses positions politiques sur sa conception philosophique; il serait plus juste de le considérer, et c'est ce que nous avons tâché de faire, comme un penseur politique anti-philosophique, donc pro-militant. On pourrait encore reprendre la distinction que font André Comte-Sponville et Luc Ferry dans $L a$ Sagesse des Modernes: Dix Questions pour Notre Temps, entre le philosophe engagé (dont la philosophie détermine explicitement son action politique) et le philosophe citoyen (dont c'est d'abord la citoyenneté, c'est-à-dire l'appartenance à une cité nécessairement divisée, qui implique sa participation).

Conclusion

23 Il nous semble important d'apporter au final la précision suivante. Ce que nous avons appelé, chez Rorty, la « séparation de la philosophie et de l'Etat » repose sur une stricte 
séparation des sphères publiques et privées. Cette séparation est d'ailleurs prévue par la Constitution des Etats-Unis. A propos de la place originellement accordée aux valeurs dans les institutions américaines, Isabelle Richet écrit: "l'Etat minimum que souhaitent les Pères fondateurs ne saurait s'occuper de former les valeurs des citoyens, et cette tâche est donc laissée aux institutions de la sphère privée, les principales à l'époque étant les Eglises [...]. Ainsi, dans leur vision, ce n'est pas le gouvernement qui produit des citoyens moraux, mais des citoyens moraux [...] qui contribuent à préserver la démocratie. La religion - et les institutions dans lesquelles elle s'incarne - conserve donc un rôle important, mais dans la sphère privée " (Richet, 103-104). Or la gauche culturelle, par des slogans du type : «tout est politique », a nui de façon considérable à l'idée même de séparation. On pourrait donc expliquer la dérive culturelle du radicalisme universitaire par une volonté de percer à jour l'idéologie dans les pratiques humaines les plus anodines: autrement dit, la gauche théorique veut réformer les personnes dans ce qu'elles ont de privé plutôt que les institutions politiques; elle veut réformer les comportements humains (d'aucuns diraient, la "nature humaine ») plutôt que les lois. Il est intéressant de voir que la droite conservatrice et religieuse a su profiter de cette brèche ouverte dans le mur de séparation du public et du privé : en effet, selon l'argumentaire fourni clés en main par certains radicaux culturels, si tout est politique, quelles raisons peut-on bien opposer à l'entrée des valeurs et des arguments religieux sur la place publique ? Voilà le genre de situation à laquelle ont conduit certaines théories de la gauche universitaire. Dans les années à venir, l'enjeu pour une gauche américaine se voulant plus militante que polémique sera de rompre avec cette rhétorique des valeurs - une rhétorique qui est maintenant utilisée de façon très efficace par la droite néo-conservatrice, au détriment de toute discussion sérieuse des politiques économiques et sociales. Cela sera une tâche extrêmement difficile, puisque cette rhétorique des valeurs ne se limite pas au débat public et à la politique intérieure, mais préside actuellement à la politique étrangère de George Bush Jr. et de son cabinet. En effet, la déclaration d'une "croisade du Bien contre le Mal», manichéenne à l'absurde, place la gauche libérale dans une position délicate, qui paraît presque paradoxale : comment faire entendre alors, dans un contexte aussi tranché, la voix d'un patriotisme américain qui ne soit pas le nationalisme militariste commandant au Moyen-Orient?

\section{BIBLIOGRAPHIE}

Barber, Benjamin, Strong Democracy (Berkeley : University of California Press, 1984).

Cheney, Lynne V., Telling the Truth (New York : Touchstone, 1996).

Fish, Stanley, There's No Such Thing As Free Speech (Oxford : Oxford University Press, 1994).

Kloppenberg, James T., The Virtues of Liberalism (Oxford : Oxford University Press, 1998).

Lasch, Christopher, The Revolt of The Elites (New York : Norton, 1996).

Transatlantica, 1 | 2008 
Laugier, Sandra, Recommencer la philosophie : la philosophie américaine aujourd'hui (Paris : Presses Universitaires de France, 1999).

Rawls, John, Political Liberalism (New York : Columbia University Press, 1996).

Richet, Isabelle, La Religion aux Etats-Unis (Paris : Presses Universitaires de France, 2001).

Rorty, Richard, Objectivity, Relativism, and Truth (Cambridge : Cambridge University Press, 1991).

------, Achieving Our Country : Leftist Thought in Twentieth-Century America (Cambridge, Mass. :

Harvard University Press, 1999).

-------, Philosophy and Social Hope (Londres : Penguin, 1999).

Roth, Philip, American Pastoral (Londres : Vintage, 1998).

\section{NOTES}

1. Pour une formulation jamesienne du pragmatisme, voir notamment « What Pragmatism Means », William James : Selected Writings (Londres : Everyman, 1995).

2. Pour une formulation deweyienne du pragmatisme, voir par exemple Reconstruction in Philosophy (New York : Dover, 2004).

3. « How is it possible that there may exist over time a stable and just society of free and equal citizens profoundly divided by reasonable religious, philosophical, and moral doctrines? » (Rawls, xxvii).

4. «The criticism from the right is that in arguing for notions like [...] the inescapability of interpretation, [...] I am undoing the fabric of civilization and opening the way to nihilist anarchy. The objection from the left is that I'm not doing that sufficiently " (Fish, 307).

5. « Conservative thinkers [...] sought to exploit public anxieties about civil rights, the New Left, and feminism. [...] When radicals challenged all cultural as well as political authority, when they defied civility and sang the praises of pleasure, when they championed drugs, sex, and subversive music, even many who considered themselves liberals began to be alarmed » (Kloppenberg, 150-151).

6. « Leftists in the academy have permitted cultural politics to supplant real politics, and have collaborated with the Right in making cultural issues central to public debate » (AOC, 14).

7. En appelant la gauche culturelle : «School of Resentment », Bloom fait bien entendu référence, non sans ironie, à Nietzsche, qui est pour elle une source majeure. Ce qui signifie que cette gauche ne semble pas procéder d'un élan, d'un enthousiasme politique, d'une volonté politique de réforme, mais bien d'une frustration, d'une réaction, et d'une volonté de destruction.

8. «[T]he Nietzscheanized left tells the country it is rotten at the core - that it is a racist, sexist, imperialist society, [...] one whose every utterance must be ruthlessly deconstructed» (PSH, 129).

9. «[...] extraordinarily self-obsessed [...], as well as absurdly over-philosophized» (PSH, 129).

10. Ancien président de l'organisation S.D.S. (Students for a Democratic Society), Todd Gitlin fait figure de repenti du radicalisme et de ses méthodes. Sa perspective, qui accueille la possibilité d'une gauche libérale et patriote, est très proche de celle Rorty. 
11. « [W]e academics marched on the English department while the Republicans took over the White House » (PSH, 260).

\section{AUTEUR}

DAVID BARRAL

ATER, Université Paris 7, Institut Charles V, david_barral@hotmail.fr 